\title{
GESTÃO E GERENCIAMENTO DE RESÍDUOS QUÍMICOS DOS LABORATÓRIOS DIDÁTICOS DO DEPARTAMENTO DE QUÍMICA E CIÊNCIAS AMBIENTAIS E DO ENTREPOSTO DE RESÍDUOS DO IBILCE/UNESP
}

Chemical Waste Management of Undergraduate Laboratories of the Department of Chemistry and Environmental Sciences and Rehabilitation of Warehouse Waste of IBILCE/UNESP

Gestión de residuos químicos de los laboratórios de enseñanza del Departamento de Química y Ciencias Ambientales y del Depósito de Residuos de IBILCE/UNESP

\author{
Clara Pires Ferreira \\ Bacharela em Química Ambiental, UNESP, Brasil. \\ ferreiraclara.pires@gmai.com \\ Mario Henrique Gonzalez \\ Professor Assistente Doutor, UNESP, Brasil. \\ gonzalez@ibilce.unesp.br \\ Luan da Silva Viana \\ Graduando em Licenciatura em Química, UNESP, Brasil. \\ luanviana1@outlook.com
}


RESUMO

Nesse estudo destaca-se a importância de um programa de gerenciamento de resíduos químicos em instituições de ensino. A quantidade de resíduos químicos gerados em aulas práticas é desprezível comparado às atividades industriais, porém o fator preocupante dessa geração é a problemática ambiental que oferecem caso não recebam tratamento e destinação finais adequados. A possibilidade da redução, reutilização e da reciclagem de resíduos favorece a disseminação de uma postura correta frente a aspectos ambientais, levando a economia de reagentes e no próprio tratamento dos resíduos gerados. A partir disso, junto à Comissão de Ética Ambiental (CEA) e o Departamento de Química e Ciências Ambientais (DQCA) do Instituto de Biociências, Letras e Ciências Exatas (IBILCE), foi trabalhado uma mudança na conduta dos alunos e pesquisadores frente à necessidade de se ter um controle sobre a produção e caracterização de resíduos, assim como proceder da forma correta na hora do descarte e identificação, através de disciplinas e eventos na busca da conscientização, na adequação dos laboratórios didáticos com normas de segurança, ensino correto de segregação de resíduos gerados nas disciplinas experimentais, criação de fichas e etiquetas de caracterização e identificação dos resíduos, respectivamente, e por fim, a revitalização do entreposto de resíduos do instituto.

PALAVRAS-CHAVE: Gerenciamento; Resíduos Químicos; Meio Ambiente.

\section{ABSTRACT}

This study highlights the importance of a chemical waste management program in educational institutions. The amount of chemical waste generated in practical classes is negligible compared to industrial activities, but the worrying factor of this generation is the environmental problems that offer unless it receives proper treatment and disposal. The possibility of reduction, reuse and recycling of waste favors the spread of a right front stance on environmental issues, leading to savings of reagents and proper treatment of waste generated. From this, by the Comissão de Ética Ambiental (CEA) and the Departamento de Química e Ciências Ambientais (DQCA) at Instituto de Biociências, Letras e Ciências Exatas (IBILCE), a change was expected in the conduct of students and researchers faced with the need of a control over the production and characterization of waste and how to proceed in the right way at the time of disposal and identification, across disciplines and events in search of awareness, adequacy of undergraduate laboratories with safety standards, correct teaching of separating waste generated in experimental disciplines, creating chips and characterization of labels and identification of waste, respectively, and finally, the revitalization of the waste warehouse.

KEYWORDS: Management; Chemical Wastes; Environment.

\section{RESUMEN}

En este estudio pone de relieve la importancia de un programa de gestión de residuos químicos en las instituciones educativas. La cantidad de residuos químicos generados en las clases prácticas es insignificante en comparación con la de las actividades industriales, sin embargo lo factor preocupante de este generación son los problemas ambientales que ofrecen si no reciben tratamiento y eliminación adecuado. La posibilidad de redución, reutilización y del reciclado de los residuos favorece la propagación de una posición para caminar derecha a los aspectos ambientales, lo que lleva a un ahorro de reactivos y el tratamiento adecuado de los residuos. A partir de este, por la Comissão de Ética Ambiental (CEA) y el Departamento de Química e Ciências Ambientais (DQCA) de lo Instituto de Biociências, Letras e Ciências Exatas (IBILCE), un cambio se trabajó en la conducta de los estudiantes y investigadores respecto a la necesidad para tener un control sobre la producción y caracterización de residuos y la forma de proceder de la manera correcta en el momento de la eliminación y localización, por medio de clases y eventos en busca de la conciencia, en la adecuación de los laboratorios de enseñanza con los estándares de seguridad, correcta enseñanza de la separación de residuos generados en las clases experimentales, la creación de chips y etiquetas de identificación y caracterización de los residuos, respectivamente, y por último, la revitalización del almacén de residuos.

PALABRAS-CLAVE: Gestión; Residuos Químicos; Medio Ambiente. 


\section{INTRODUÇÃO}

As atividades químicas repetidamente vêm sido associadas, direta ou indiretamente, à grande parte dos "acidentes ambientais", mesmo que algumas atividades humanas também contribuam para a degradação e poluição ambientais. Assim, a necessidade do desenvolvimento de novas condutas e métodos mais sustentáveis, como a Química Verde com princípio de reduzir, reutilizar ou reciclar os resíduos gerados - tem sido amplamente discutida neste intuito'.

As universidades que possuem cursos nas áreas de Química e afins têm sido relacionadas com o problema a respeito do tratamento e à disposição final dos resíduos gerados em laboratórios, tanto de ensino quanto de pesquisa ${ }^{2}$. O trabalho de conscientização referente a produção responsável, onde atribui ao gerador do resíduo a responsabilidade para identificar, tratar quando possível e dispor de forma segura seu resíduo, tem como objetivo estimular a preservação ambiental e mostrar a necessidade da implantação de programas de gestão e gerenciamento de resíduos químicos. ${ }^{3}$

Os resíduos são resultantes de processos das mais diversas atividades industrial, doméstica, hospitalar, comercial, agrícola, entre outros, podendo ser nos estados sólido, líquido ou gasoso ${ }^{4}$. Sendo mais específico, os resíduos químicos são aqueles produtos que não podem ter tratamento convencional, e têm potencial de causar danos a organismos vivos, materiais, estruturas ou ao meio ambiente; ou ainda, podendo oferecer risco e perigo quando interage com outros materiais ${ }^{5}$.

Com o desenvolvimento da humanidade, principalmente após a revolução industrial, houve um aumento da produção de resíduos, consequentemente, aumentou-se a preocupação ambiental com o impacto que estes resíduos poderiam causar. Dessa forma, atitudes foram tomadas, como as suas classificações e a criação de legislações e normas técnicas, para a resolução do problema. Atualmente, algumas das mais conceituadas instituições do Brasil, tendo em mente reverter esse quadro problemático dos resíduos, colocam em prática a Responsabilidade Objetiva da Lei 6.938/81 da Política Nacional do Meio Ambiente, em que o gerador dos resíduos torna-se responsável por eles e pelos danos causados ao serem descartados no ambiente ${ }^{6}$. Os resíduos apresentam classificação de acordo com a norma da ABNT NBR 10.004 ${ }^{7}$, onde são classificados em:

- Resíduos classe I - PERIGOSOS: aqueles que possuem características de inflamabilidade, corrosividade, reatividade, toxicidade ou patogenicidade;

- Resíduos classe II - NÃO PERIGOSOS:

$\checkmark$ Resíduos classe II A - não inertes: aqueles que não apresentam periculosidade;

$\checkmark$ Resíduos classe II B - inertes: aqueles que quando submetidos aos testes de solubilização (ABNT/NBR 10.007) não afetam a potabilidade da água. Não se degradam ou se decompõe quando dispostos no solo e muitos deles são recicláveis.

No campus de São José do Rio Preto, o gerenciamento dos resíduos químicos é realizado pela Comissão de Ética Ambiental (CEA) desde 2004 e tem como base, o Programa de 
Gerenciamento de Resíduos - PGR da UNESP ${ }^{8}$, instituído em agosto de 2006. Há a necessidade de trazer diretrizes básicas para a gestão e gerenciamento dos resíduos para uma unidade geradora $(U G)^{9}$. Em termos organizacionais os passos de hierarquia para implementação de um programa de gerenciamento de resíduos é apresentado na Figura 1.

Figura 1. Fluxograma sobre a hierarquia de um Programa de Gerenciamento de Resíduos.

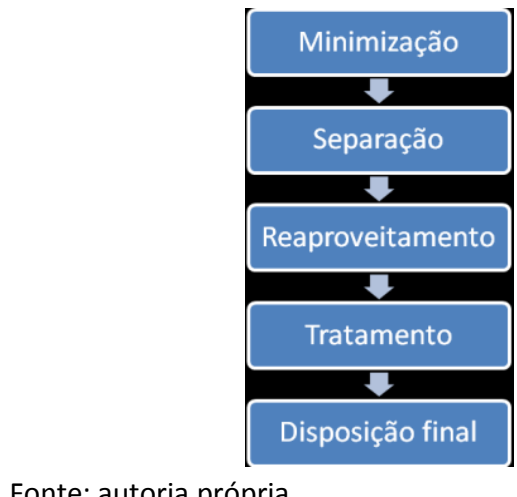

O laboratório de química é um local que fornece conhecimentos práticos e enriquecedores para o futuro profissional desta área e outras áreas ligadas, contudo, é um local que oferece perigos caso não utilizado com o devido cuidado, pois expõe os estudantes à substâncias corrosivas, tóxicas, inflamáveis, etc, sendo essencial o conhecimento e acesso às normas de segurança. No almoxarifado dos laboratórios são armazenados produtos químicos de diferentes fases ou composições, por esse motivo é necessário se ter um cuidado redobrado na sua estocagem ${ }^{10}$. O conhecimento do Mapa de Risco de um laboratório é essencial para todos os indivíduos que tem contato com este local. Neste documento, expõem-se os riscos como físicos, químicos, biológicos, ergonômicos e de acidentes e é elaborado pela CIPA (Comissão Interna de Prevenção de Acidentes) ${ }^{11}$.

\section{OBJETIVOS}

Com este trabalho buscou-se a atuação sustentável que englobasse a política de gestão de resíduos em todas as suas etapas, conscientizando todos os envolvidos no processo de produção de resíduos, além de realizar o enquadramento destes resíduos segundo as legislações vigentes.

\section{METODOLOGIA}

Um programa de gerenciamento deve sempre adotar a regra da responsabilidade objetiva, ou seja, quem gerou o resíduo é responsável pelo mesmo, e praticar sempre a seguinte hierarquia de atividades:

1- Prevenção na geração de resíduos (perigosos ou não);

2- Minimizar a proporção de resíduos perigosos que são inevitavelmente gerados; 
3- Segregar e concentrar correntes de resíduos de modo a tornar viável e economicamente possível a atividade gerenciadora;

4- Reuso interno ou externo;

5- Reciclar o componente material ou energético do resíduo;

6- Manter todo resíduo produzido na sua forma mais passível de tratamento;

7- Tratar e dispor o resíduo de maneira segura. ${ }^{12}$

O trabalho de conscientização é a etapa mais importante, e foi realizado visando orientar os alunos envolvidos direta e indiretamente com a produção e manipulação de resíduos, sobre o destino ideal para o descarte e incentivando a atuação deles como multiplicadores de informação. Para efetivar a consciência ética da manipulação de resíduos e sua importância, atividades de ensino foram propostas e realizadas, tal como a criação de uma disciplina optativa de Gestão e Gerenciamento de Resíduos Químicos para os alunos de graduação em Química e um evento, o I Workshop de Segurança e Gerenciamento de Resíduos Químicos, abrangendo alunos de graduação, pós-graduação, profissionais da área e comunidade externa. Para os discentes dos cursos de graduação em química e das áreas que utilizam os laboratórios didáticos a criação de material didático para o auxílio dos usuários do laboratório didático foi confeccionado, em que consiste de:

- Pasta composta de 72 fichas de segurança dos produtos químicos (FISPQ) mais comumente empregados nas práticas, tendo os alunos livre acesso a pasta para o conhecimento da segurança química e periculosidade dos reagentes e solventes utilizados durante as aulas experimentais;

- Impressão e disponibilidade de etiquetas de identificação para os frascos dos resíduos, de acordo com o modelo da UNESP, em material adesivo;

- Pasta única contendo um manual para o preenchimento correto do Diagrama de Hommel;

- Informações de boas práticas de laboratório, manual de EPIs e EPCs, lista de incompatibilidade e listagem de substâncias que podem ser descartadas na pia e no lixo.

Todas as atividades no laboratório devem apresentar condições ideais de segurança, já que um procedimento indevido pode causar acidentes de pequenas a grandes magnitudes, trazendo sérios danos a todos os presentes no local, como também, proporcionar o escoamento de substâncias que, em contato com matrizes ambientais (água, ar, solo) ocasionam contaminações. Para ambientes de trabalho, em que se situam produtos químicos, existem normas específicas para prevenção de acidentes do trabalho como, Boas Práticas de Laboratório e Sinalização de Segurança - NR 26, dentre outras a partir da Portaria 3.214, de 08/06/1978 ${ }^{13}$. Providenciar segurança dentro do laboratório também promove a qualidade do trabalho daquele local. Sendo assim, a fim de evitar tais problemas, a adaptação dos laboratórios didáticos se deu através de faixas de segurança e delimitação da área do chuveiro e lava-olhos com fita antiderrapante; placas de sinalização de extintores e saídas de emergência que antes eram inexistentes; além do material didático fornecido aos discentes, já mencionado anteriormente, que se localizam em locais de fácil e rápido acesso à consulta. A 
divulgação do programa de gerenciamento de resíduos químicos também fora feita através da participação e apresentação de trabalho em eventos científicos.

\section{RESULTADOS}

As atividades foram iniciadas com a explicação sobre o funcionamento de um Programa de Gerenciamento de Resíduos Químicos no laboratório didático. O sucesso de um PGR se baseia em diversos fatores, extremamente importantes, como o treinamento dos usuários do laboratório, divulgação do programa em todas suas etapas e qualquer tipo de ação educacional. Assim, criar a consciência da importância dos resíduos químicos nos discentes é fundamental para o crescimento do programa. Espera-se que os discentes que tiverem a vivência nos tópicos de gestão e gerenciamento de resíduos químicos, passem a cobrar de seus docentes e funcionários, tornando um ato cotidiano em suas atividades laboratoriais, podendo expandir para seus trabalhos de pesquisa. Para isso, fora criado um fluxograma (Fig. 2) do correto gerenciamento de resíduos químicos durante as aulas práticas. Vale lembrar que essa é uma proposta, ou seja, essas etapas poderão ser seguidas ou não. No início do semestre o aluno deverá ser informado sobre estas etapas e como proceder no caso do uso dos reagentes e na produção dos resíduos químicos.

Figura 2. Fluxograma proposto para o gerenciamento de resíduos químicos nas aulas práticas.

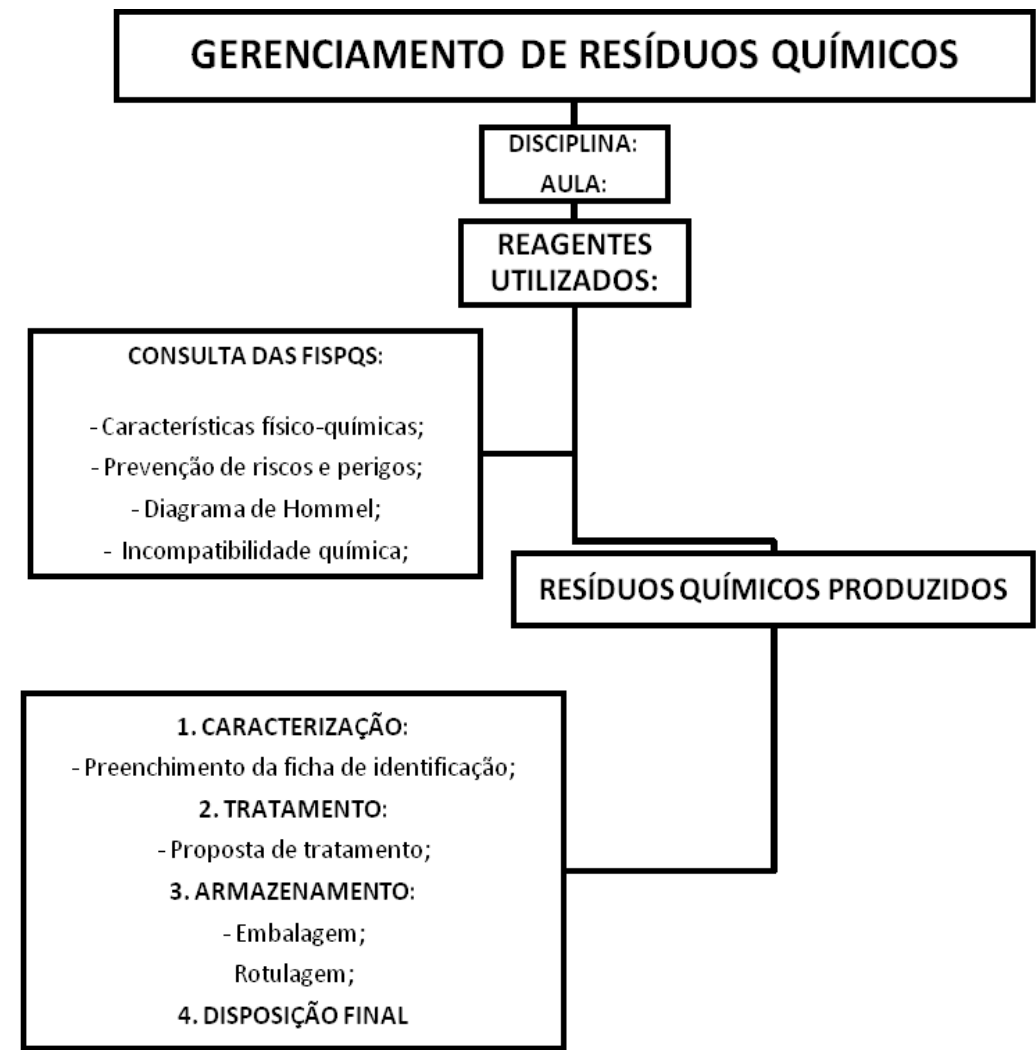

Fonte: autoria própria. 
Com base no fluxograma, a criação do material didático desenvolvido tornar-se-á um trabalho usual antes, durante e depois das aulas que gerarem resíduos químicos, estando à disposição para consulta dos discentes durante suas aulas práticas. $\mathrm{O}$ objetivo deste material foi facilitar e melhorar o entendimento a respeito do correto gerenciamento de resíduos produzidos no laboratório, bem como as normas de segurança. A ideia foi fazer com que todo resíduo produzido, caso não tenha tratamento prévio ou descarte imediato, possa ser identificado corretamente (Fig.3) e rotulado de acordo com as normas da UNESP. O principal intuito com estes trabalhos de conscientização conjuntamente com o material didático foi para que os discentes pudessem utilizar seus aprendizados da graduação realizando uma proposta de identificação e tratamento, baseando-se em suas características, além de ter conhecimento dos perigos e riscos que podem ocorrer de uma atividade da qual ele mesmo foi responsável pela produção do resíduo químico.

Figura 3. Modelo de ficha proposta para caracterização e armazenagem de resíduos químicos.

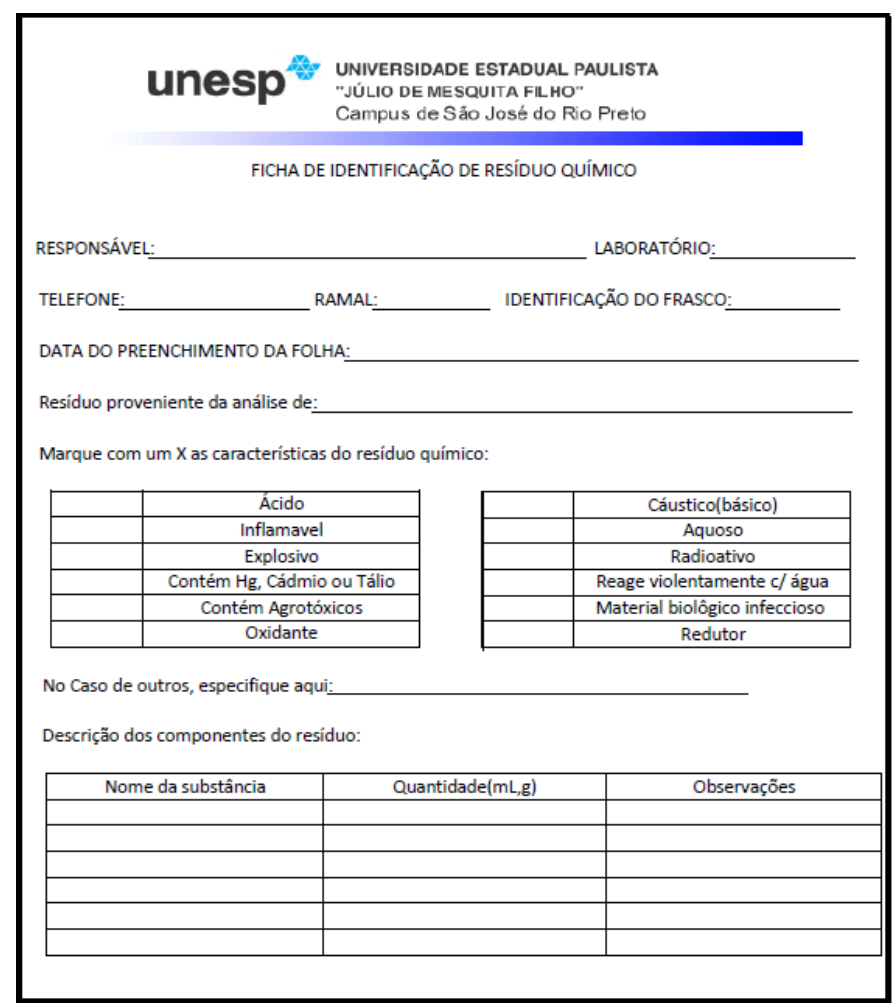

Fonte: autoria própria.

Todas as atividades no laboratório devem apresentar condições ideais de segurança, já que um procedimento indevido pode causar acidentes de pequenas a grandes magnitudes. A fim de evitar tais problemas, a adaptação dos laboratórios didáticos se deu através da aplicação de faixas de segurança e delimitação da área do chuveiro e lava-olhos com fitas anti-derrapantes; placas de sinalização de extintores e saídas de emergência; listagem de equipamentos de proteção individual e coletiva; modelos do preenchimento do diagrama de Hommel (Fig. 4); disponibilização de FISPQs de substâncias comumente utilizadas em aulas e quadro de incompatibilidade química. 
Figura 4. Diagrama de Hommel e Guia para os Códigos de NFPA.
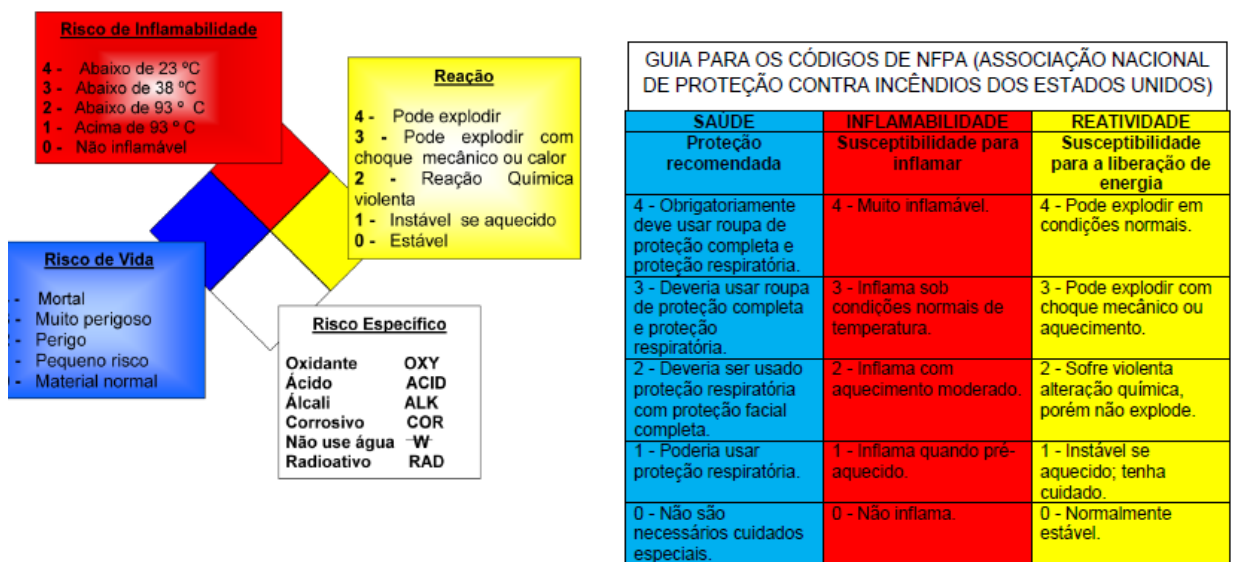

Fonte: adaptação <http://www.qca.ibilce.unesp.br/prevencao/classificacaonfpa.pdf>

Como forma de divulgação e conscientização do programa de gerenciamento de resíduos, no 10 semestre do ano letivo de 2015, ofertou-se a disciplina optativa Gestão e Gerenciamento de Resíduos Químicos para a graduação em Química, tendo se matriculado 42 discentes. A ementa baseou-se no ideal PGR ilustrado na literatura, de acordo com instituições de ensino superior e outros que obtiveram sucesso na implantação do programa. Aos alunos eram passados conceitos teóricos a respeito das etapas hierárquicas do PGR, bem como atividades em que se mostrou a grande quantidade de resíduos produzidos em diversas áreas e setores. O interesse foi tão grande, que resultou em bons trabalhos, em forma de avaliação de seminários e monografias em diversas áreas de resíduos em geral.

Em busca da divulgação do programa e trabalho realizado, no dia 12 de agosto de 2015 foi realizado, com a ajuda da Empresa Júnior QUANTIS - Tecnologia e Sustentabilidade o I Workshop de Segurança e Gerenciamento de Resíduos Químicos, que contou com a presença de 91 inscritos (dentre estes, discentes de graduação e pós-graduação, bem como profissionais da área), trazendo palestras e atividades práticas do tema, dentre eles:

1) Atendimento a Emergências Químicas - CETESB;

2) Programa de Gerenciamento de Resíduos da UNESP (PGR);

3) Programa de Gestão de Resíduos da Embrapa Pecuária Sudeste;

4) Atividade Prática: Atendimento a Emergências Químicas Envolvendo Produtos Perigosos;

5) Atividade Prática: Visita ao laboratório didático e Entreposto de Resíduos do IBILCE/UNESP.

Este evento será bienal a partir de sua primeira edição.

Os resíduos químicos dos laboratórios da UNESP são armazenados em um entreposto que responde as condições básicas de segurança como pavimento vazado, separação de inflamáveis e não inflamáveis, caixa de contenção, afastamento adequado de outras construções, acesso facilitado para combate a incêndios, instalações elétricas adequadas, equipamento de combate a incêndios, com as devidas identificações. Quando o entreposto 
está com sua capacidade de armazenamento no máximo é feita uma solicitação para uma empresa de tratamento de resíduos (escolhida pela UNESP seguindo os procedimentos de seleção) venha recolher esses resíduos e lhes dê os corretos destinos. Em 2015, a empresa responsável pelo transporte e disposição final dos resíduos retirou, aproximadamente, duas toneladas de resíduo armazenado. A preocupação que se teve, a princípio, foi de que muitos estavam incorretamente identificados, ou em frascos inadequados, além de estarem dispostos sem seguir as regras de incompatibilidade química, tornando aquele local perigoso.

Com a retirada de grande parte dos resíduos estocados, ainda havia uma grande quantidade que necessitava ser disposta adequadamente, então no mês de julho de 2015 executou-se a limpeza a seco do entreposto, já que a quantidade de poeira era grande, o que poderia resultar em algum acidente. Na Figura 5 é possível comparar as mudanças no local antes e depois da limpeza.

Figura 5. Entreposto de Armazenamento de Resíduos Químicos do IBILCE/UNESP antes e depois da limpeza no mês de julho de 2015.

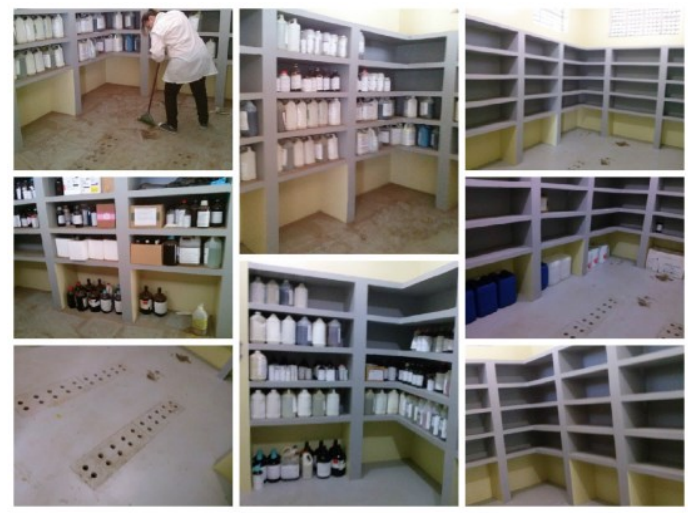

Fonte: autoria própria.

Após a coleta de resíduos do ano de 2015, realizou-se um dia para a transferência dos resíduos dos laboratórios produtores (Unidades Geradoras) para o entreposto de resíduo. Atualmente há por volta de 1,5 tonelada de passivo ambiental estocado no entreposto.

Os resíduos foram separados por um sistema de cores nas prateleiras de acordo com suas características, ou seja, agrupados em orgânicos (vermelho), inorgânicos (verde), biológicos (amarelo), sólidos (marrom) e sem identificação (azul). Esse sistema de cores facilita na visualização para a correta armazenagem dos resíduos. Com essas medidas, foi realizado um levantamento de todo o resíduo armazenado e quantidade dele, relacionando-o sempre a seu responsável gerador, para que se tenha controle na retirada pela empresa responsável.

\section{CONCLUSÃO}

O Programa de Gerenciamento de Resíduos no Campus do IBILCE é promissor e além de viável. Com o grande impacto a respeito da educação ambiental, este programa dá oportunidade de treinamento aos discentes a formação de agentes multiplicadores da hierarquia de resíduos, permitindo que o profissional egresso, independente de sua formação, adquira uma 
consciência ética com relação ao uso e descarte de produtos visando a preservação ambiental. O sucesso do projeto depende da atuação responsável de professores, alunos e funcionários dos laboratórios que envolvem atividades químicas.

Os resultados obtidos são prévios, pelo fato de esta trabalho ser de médio a longo prazo, porém o programa encontra-se ainda em fase de implementação, tendo que realizar melhorias e inovações que possam atrair e facilitar as atividades de todos os geradores de resíduos do campus. A perspectiva é contribuir para a solução definitiva da disposição, tratamento, recuperação e reaproveitamento, além do descarte adequado dos resíduos químicos gerados (sólidos, líquidos e gasosos) nos muitos laboratórios, tanto de ensino e pesquisa.

\section{AGRADECIMENTO}

A Universidade Estadual Paulista "Júlio de Mesquita Filho", Edital № 68/2014 - PRAD/UNESP: Gestão de Resíduos Químicos: Revitalização do Programa de Gerenciamento de Resíduos Químicos dos Laboratórios de Ensino do Departamento de Química e Ciências Ambientais do IBILCE em São José do Rio Preto, ao IBILCE/Instituto de Biociências Letras e Ciências Exatas e Departamento de Química e Ciências Ambientais e a FAPERP - Fundação de Apoio à Pesquisa e Extensão de São José do Rio Preto pelo auxílio concedido (Processo $n^{\circ}$ 77/2015) na organização do "I Workshop de Segurança e Gerenciamento de Resíduos Químicos".

\section{REFERÊNCIAS BIBLIOGRÁFICAS}

'LENARDÃO, E. J.; FREITAG, R. A.; DABdouB, M. J.; BATISTA, A. C. F.; SILVEIRA, C. C. "Green chemistry" - Os 12 princípios da química verde e sua inserção nas atividades de ensino e pesquisa. Química Nova, vol. 26, no. 1, São Paulo, Jan-Fev/2003. Disponível em: <http://www.scielo.br/scielo.php?pid=S010040422003000100020\&script=sci_arttext>. Acesso: 23 de agosto 2015.

${ }^{2}$ GERBASE, A. E.; COELHO, F. S.; MACHADO, P. F. L.; FERREIRA, V. F. Gerenciamento de Resíduos em Instituições de Ensino e Pesquisa. Química Nova, vol. 28, no. 1, São Paulo, Jan-Fev/2005. Disponível em: <http://www.scielo.br/scielo.php?pid=S010040422005000100001\&script=sci_arttext>. Acesso: 23 de agosto de 2015.

${ }^{3}$ ALECRIM, G. F.; MAGNO, K. S.; MENDONÇA, R. B. S.; VALLE, C. M. Gerenciamento dos Resíduos Gerados nas Disciplinas Química Geral e Química Inorgânica dos Cursos da Área de Química do CEFET-AM. II Congresso de Pesquisa e Inovação na Rede Norte Nordeste de Educação Tecnológica, João Pessoa, 2007. Disponível em: < http://www.redenet.edu.br/publicacoes/arquivos/20080213_092225_MElO-090.pdf>. Acesso: 23 de agosto de 2015.

${ }^{4}$ CAVALCANTI, J. E. A década de 90 é dos resíduos sólidos. Revista Saneamento Ambiental, n. 54, p. 16-24, nov-dez 1998. Disponível em: <http://riosvivos.org.br/a/Noticia/A+decada+de+90+e+dos+residuos+solidos/3223>. Acesso em: 22 de abril 2016.

${ }^{5}$ Descarte de produtos e resíduos químicos. Disponível em: <http://www.fragmaq.com.br/blog/reciclagemresiduos-liquidos/descarte-de-produtos-e-residuos-quimicos/>. Acesso em: 22 de abril de 2016.

${ }^{6}$ NOLASCO, F.R.; TAVARES, G.A.; BENDASSOLLI, J.A. Implantação de Programas de Gerenciamento de Resíduos Químicos Laboratoriais em universidades: análise crítica e recomendações. Eng. Sanitária e Ambiental, vol.11, no.2, Rio de Janeiro, Abr-Jun/2006. Disponível em: 
<http://www.scielo.br/scielo.php?script=sci_arttext\&pid=S1413-41522006000200004>. Acesso em: 22 de abril de 2016.

7Home Page: ASSOCIAÇÃO BRASILEIRA DE NORMAS TÉCNICAS (ABNT), NBR 10.004, 1987 b. Resíduos Sólidos Classificação. Rio de Janeiro: ABNT, 2004.

${ }^{8}$ Programa de Gerenciamento de Resíduos da Unesp. Disponível em: <http://www.unesp.br/portal\#!/pgr/apresentacao/>. Acesso: 07 de dezembro de 2013.

${ }^{11}$ JARDIM, W. F. Gerenciamento de Resíduos Químicos em Laboratórios de Ensino e Pesquisa. Química Nova, vol. 21, p. 671, São Paulo, 1998. Disponível em: < http://www.scielo.br/pdf/qn/v21n5/2943.pdf>. Acesso: 23 de agosto de 2015.

${ }^{10}$ ALBERGUINI, L.B.A.; SILVA, L.C.; REZENDE, M.O.O. Tratamento de Resíduos Químicos - Guia prático para a solução de resíduos químicos. Ed. RiMa, 2005.

${ }^{11}$ Comissão de Riscos Químicos - Unifal. EQUIPAMENTOS DE PROTEÇÃo INDIVIDUAL (EPI) E SUAS UTILIDADES NOS LABORATÓRIO. Disponível em: http://www.unifal-mg.edu.br/riscosquimicos/epis > acesso em 8/12/2013.

${ }^{12}$ ALBERGUINI, L.B.A.; SILVA, L.C.; REZENDE, M.O.O.R. Laboratório de resíduos químicos do campus USP - São Carlos - resultados da experiência pioneira em gestão e gerenciamento de resíduos químicos em um campus universitário. Química Nova, vol. 26, no. 02, São Paulo, Março-Abril/2003. Disponível em: <http://www.scielo.br/scielo.php?script=sci_arttext\&pid=S0100-40422003000200026>. Acesso em: 05 de setembro de 2015.

${ }^{13}$ PORTARIA GM n.o 3.214, de 08 de junho de 1978 - NR 26 - Sinalização de Segurança. Disponível em: <http://www.guiatrabalhista.com.br/legislacao/nr/nr26.htm>. Acesso em: 05 de setembro de 2015. 